\title{
Performance quantification of Wireless Sensor Networks by implementing ZRP
}

\author{
Barjinder Singh \\ Research fellow,M.Tech,Dept. of CSE \\ R.I.E.T Phagwara \\ barjinder_jal@yahoo.co.in
}

\begin{abstract}
A sensor network is composed of a large number of autonomous sensor nodes, which are densely deployed in the area of interest i.e either inside the phenomenon or very close to it. Routing is an important operation, being the foundation of data exchanging between wireless devices.Zone Routing Protocol was the first hybrid routing protocol with both a proactive and a reactive routing component. $Z R P$ was proposed to reduce the control overhead of proactive routing protocols and decrease the latency caused by route discovery in reactive routing protocols. ZRP defines a zone around each node consisting of the numbers of neighbourhood. During my research work, I applied the hybrid routing protocol in wireless sensors and measure the corresponding performance of the network in terms of overhead, delay and throughput.[10]
\end{abstract}

Keywords:-Sensors, Zones, wireless sensor network , ZRP, IARP, IERP, Zone Radius, Proactive, Reactive, Hybrid Routing,Intrazone,Interzone.

\section{Introduction}

It consists of a set of small devices with sensing and wireless communication capabilities. Those small devices are named sensor nodes, and are deployed within a special area to monitor a physical phenomenon. E.g: Anthrax, Motes etc. WSN devices have severe resource constraints in terms of energy, computation and memory. An illustration is given in Figure1.[3]

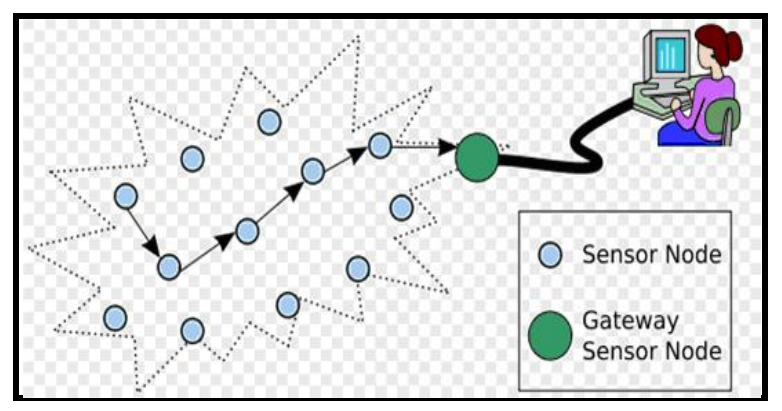

Figure1: Sensor nodes,topology and communication

Wireless sensors sense the information, then all the data is collected by the gateway sensor node such as a sink node,

\author{
Er. Rishma, Assistant Professor \\ Dept. of CSE \\ R.I.E.T Phagwara \\ rishma_chawla_2004@yahoo.com
}

which further relay it to the server where data analysis is performed. Today, it has a very wide range of applications such as environment monitoring, forest fire detection, landslide detection, greenhouse monitoring etc

Routing is the process of selecting optimal pathway over the network to route the traffic. In case of Table Driven Routing Protocol or Proactive routing each node maintains one or more tables containing routing information to every other node in the network. Tables need to be consistent and up-to-date view of the network. Updates propagate through the network. E.g : DSDV,OLSR,WRP etc. [12,13]

On the other hand, Source Initiated On demand routing protocol is Reactive in nature. It uses on-demand style, and create routes only when it is desired by the source node. When a node requires a route to a destination, it initiates a route discovery process. Route is maintained until destination becomes unreachable, or source no longer is interested in destination. E.g: AODV,DSR etc.

ZRP is a hybrid[13] protocol that incorporates the merits of on-demand and proactive routing protocol, which provides efficient and fast discovery of route. It limits the scope of the proactive procedure only to the node's local neighbourhood. So in such a way ZRP protocol reduces the waste associated with routing update traffic of proactive routing to the limited number of zone members. On the other hand, performance and throughput becomes efficient as the querying is performed on selected nodes in the network, rather than flooding queries all over the network.

\section{Proactive and Reactive Routing}

A reactive routing protocol tries to find a route from $S$ to $D$ only on-demand i.e., when the route is required, for example, DSR and AODV are such protocols. The main advantage of a reactive protocol is the low overhead of control messages. However, reactive protocols have higher latency in discovering routes. A reactive routing protocol tries to find a route from $\mathrm{S}$ to D only on-demand i.e., when the route is required, for example, DSR and AODV are such protocols. The main advantage of a reactive protocol is the low overhead of control messages. However, reactive protocols have higher latency in discovering routes. [4] 
It is possible to exploit the good features of both reactive and proactive protcols and the Zone routing protocol does that. The proactive part of the protocol is restricted to a small neighbourhood of a node and the reactive part is used for routing across the network. This reduces latency in route discovery and reduces the number of control messages as well.

Each node $\mathrm{S}$ in the network has a routing zone. This is the proactive zone for $\mathrm{S}$ as $\mathrm{S}$ collects information about its routing zone in the manner of the DSDV protocol. If the radius of the routing zone is "r", each node in the zone can be reached within $r$ hops from $S$. The minimum distance of a peripheral node from $\mathrm{S}$ is $\mathrm{r}$ (the radius). All nodes except $\mathrm{L}$ are in the routing zone of $S$ with radius 2. (illustrated in Figure2) [4]

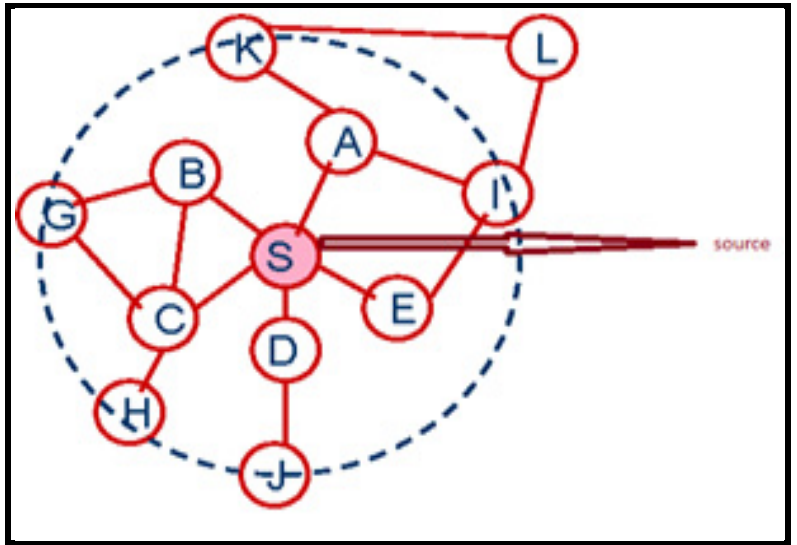

Figure2:sensor nodes with in and outside Zone

The routing in ZRP is divided into two parts :

2.1 Intrazone routing : It is defined as the routing where the packet is sent within the routing zone of the source node to reach the peripheral nodes. intra-zone protocol is a proactive routing protocol. IARP is used inside routing zones. A route to a destination within the local zone can be established from the source's proactively cached routing table by IARP. $[1,4]$

2.2 Interzone routing : It is defined as the routing Where the packet is sent from the peripheral nodes towards the destination node. IntErzone Routing Protocol (IERP) is a global reactive routing component of ZRP. It determine the proper route only when required (on-demand). [1,4]

Each node collects information about all the nodes in its routing zone proactively. This strategy is similar to a proactive protocol like DSDV. Each node maintains a route table for its routing zone, so that it can find a route to any node in the routing zone from this table. In the original ZRP proposal, intrazone routing is done by maintaining a link state table at each node. Each node periodically broadcasts a message similar to a hello message. We call this message as a zone notification message.

Suppose the zone radius is $r$ for $r>1$. A hello message dies after one hop, i.e., after reaching a node's neighbours. A zone notification mesage dies after " $r$ " hops, i.e., after reaching the node's neighbours at a distance of "r" hops.

Each node receiving this message decreases the hop count of the message by 1 and forwards the message to its neighbours. The message is not forwarded any more when the hop count is 0 . Each node $P$ keeps track of its neighbour Q from whom it received the message through an entry in its link state table. $\mathrm{P}$ can keep track of all the nodes in its routing zone through its link state table.

The interzone routing discovers routes to the destination reactively. Consider a source $(S)$ and a destination (D). If D is within the routing zone of $S$, the routing is completed in the intrazone routing phase. Otherwise, $S$ sends the packet to the peripheral nodes of its zone through bordercasting.

The bordercasting to peripheral nodes can be done mainly in two ways : By maintaining a multicast tree for the peripheral nodes. $\mathrm{S}$ is the root of this tree. Otherwise, $\mathrm{S}$ maintains complete routing table for its zone and routes the packet to the peripheral nodes by consulting this routing table. S sends a route request (RREQ) message to the peripheral nodes of its zone through bordercasting. (illustrated in Figure4) [14,15]

Each peripheral node $\mathrm{P}$ executes the same algorithm. First, $\mathrm{P}$ checks whether the destination $\mathrm{D}$ is within its routing zone and if so, sends the packet to D. Otherwise, $\mathrm{P}$ sends the packet to the peripheral nodes of its routing zone through bordercasting. (illustrated in Figure3)

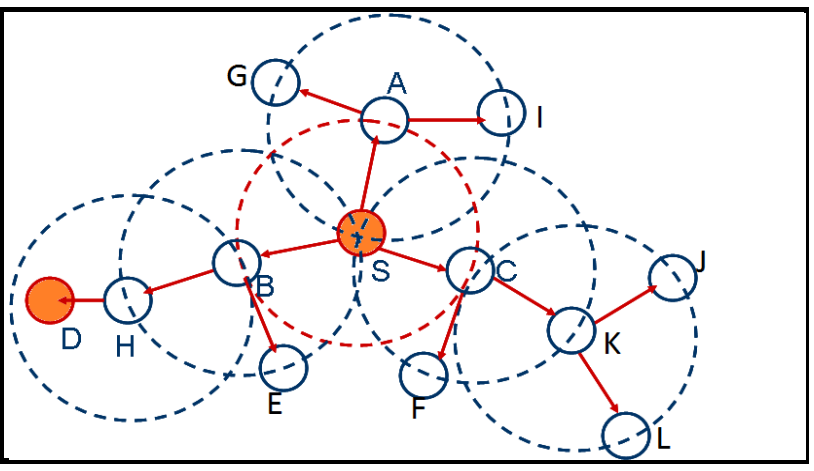

Figure3: source to destination communication through various nodes[2]

If a node $\mathrm{P}$ finds that the destination $\mathrm{D}$ is within its routing zone, $\mathrm{P}$ can initiate a route reply. Each node appends its address to the RREQ message during the route request phase. This is similar to route request phase in DSR. This accumulated address can be used to send the route reply (RREP) back to the source node S. (ilustrated in Figure5).

An alternative strategy is to keep forward and backward links at every node's route table similar to the AODV protocol. This helps in keeping the packet size constant. A RREQ usually results in more than one RREP and ZRP keeps track of more than one path between $S$ and $D$. An alternative path is chosen in case one path is broken. 
ZRP: Example with Zone Radius $=r=2$

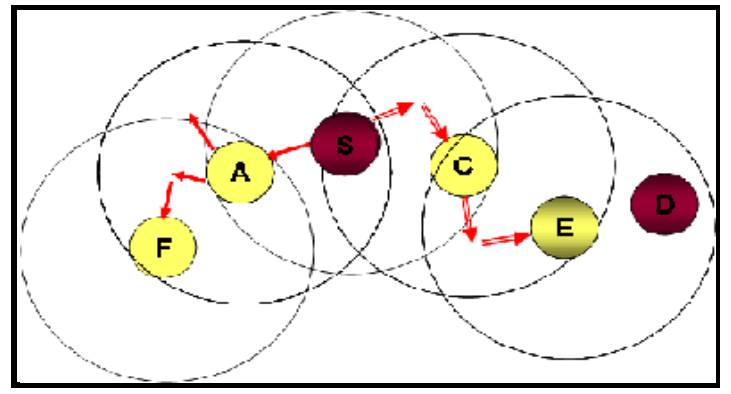

Figure4: Route Request[2,7]

Denotes route request, $\mathrm{S}$ performs route discovery for $\mathrm{D}$

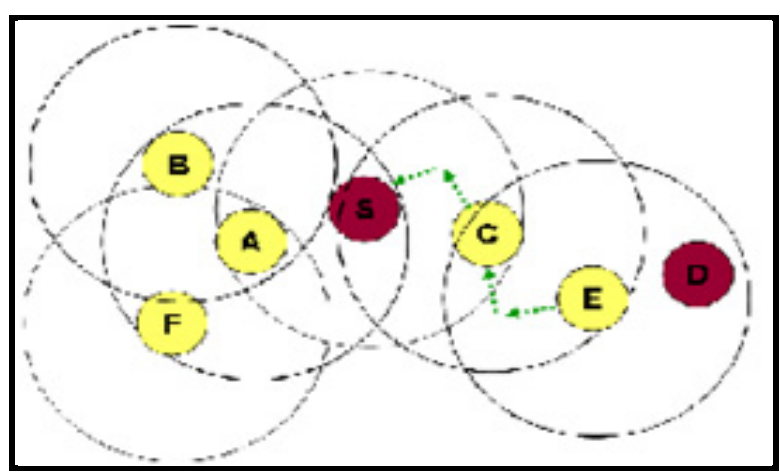

Figure5: Route Reply[2,7]

- - -> Denotes route reply

As illustrated in Figure5, E knows route from E to D, so route request need not to be farwarded to $\mathrm{D}$ from $\mathrm{E}$.

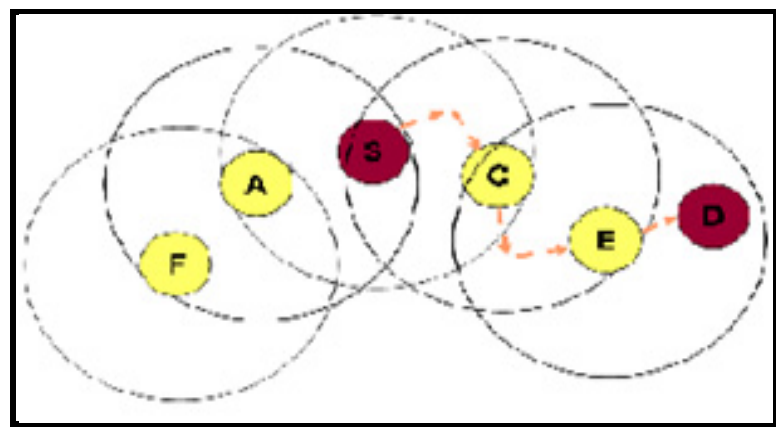

Figure6: Data Transfer[2]

- -> Denotes route taken by data

After collecting all the information regarding the desination , the Source send all the packets and the packets follow the discovered route.(conspicuous in Figure6)

\section{Architecture of ZRP}

The Zone Routing Protocol, as its name implies, is based on the concept of zones. A routing zone is defined for each node separately, and the zones of neighboring nodes overlap. The routing zone has a radius " $r$ " expressed in hops. The zone thus includes the nodes, whose distance from the node in question is at most $r$ hops.

The relationship between the components is illustrated in Figure7. Route updates are triggered by NDP, which notifies IARP [5] when the neighbor table is updated. IERP [5] uses the routing table of IARP to respond to route queries. IERP forwards queries with BRP. BRP[4] uses the routing table of IARP to guide route queries away from the query source.

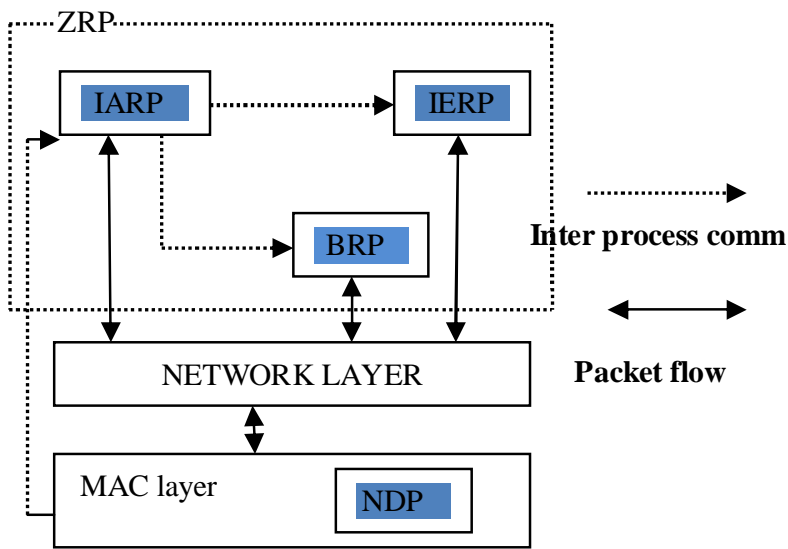

Figure7: ZRP architecture[3]

In order to detect new neighbour nodes and link failures, the ZRP relies on a Neighbour Discovery Protocol (NDP) provided by the Media Access Control (MAC) layer. NDP transmits "HELLO" beacons at regular intervals. Upon receiving a beacon, the neighbour table is updated. Neighbours, for which no beacon has been received within a specified time, are removed from the table. If the MAC layer does not include a NDP, the functionality must be provided by IARP.[4]

\section{Proposed Algorithm}

(1) First of all Obtain the co-ordinate position of complete deployment area.

(2) Then Compute the deployment area. Divide the deployment area into $\mathrm{n}$ equal no. of zones.

(3) Obtain the co-ordinate point for each sensor node with in the deployment area using hello packets.

(4) Now based on the co-ordinate positions of sensor nodes divide the deployment area into zones in a way that the concentration of a sensor nodes with in each zone should be approximately same. (may vary also) 
(5) Associate each node-id with its zone-id. Each node with in the zone will now send hello packets \& form communication link with the neighbors found in its zone.

(6) From this step we will obtain a mesh out of which we can obtain a best tree for routing packets from source to destination.(the best tree can be obtained using min spanning tree).

(7) Compute the energy[6] level of each node with in the zone.

(8) Based on the energy level the nodes with the highest energy level will now act as root of the tree. The root node will act as sink node. $[8,9]$

(9) This sink node will aggregate the data for a limited time period. After the time period (T0), the sink node will shift the load to its either of the child(prefer BFS or DFS).

(10) Compute the energy level of each sink node with in the deployment area and obtain mesh(graph) among them from which we can farther obtain a tree structure as in the previous steps.

(11) The root node of the tree obtained from sink node will now communicate or form a link with the base station.

\subsection{Intrazone routing protocol Algorithm and steps:}

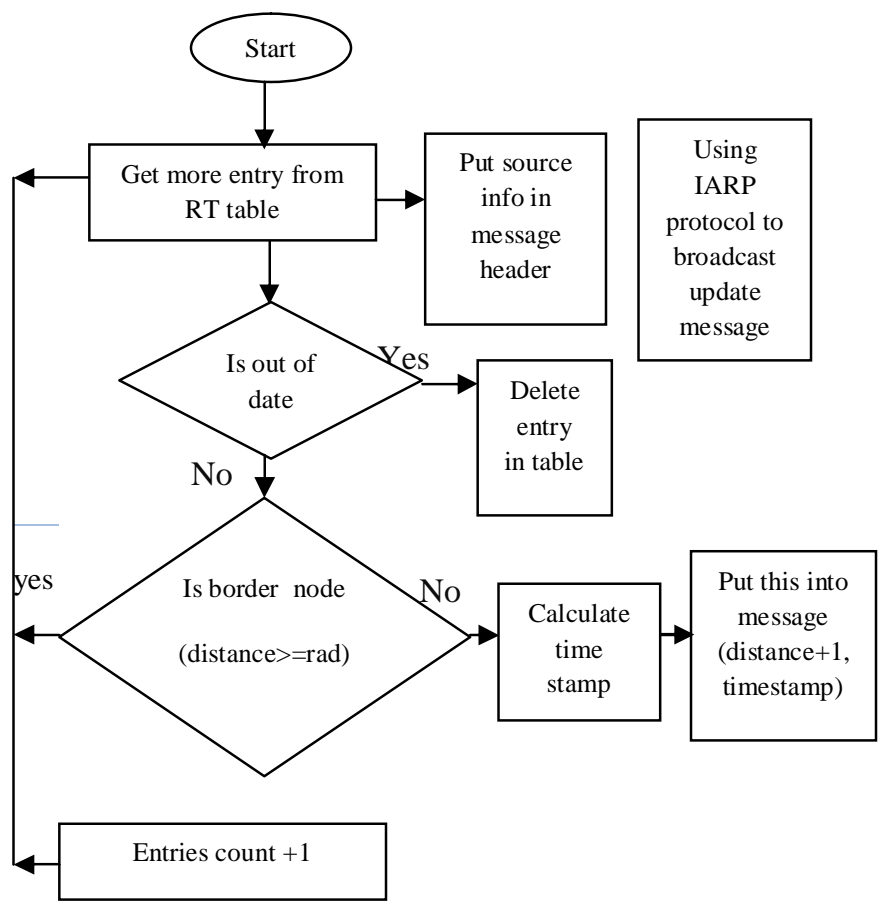

Figure8: IARP message sending part 1
1) Create nodes and network between them

2) Initialize them and start network.

3) Send packet from one node to another.

4) Receiving node extract packet and then load.

(1) Extract IARP packet to following variables: $\{$ dest , mask, next_hop_1, next_hop_2, route metric,hop_count \}

(2) Load packet: load values of the aforementioned variables into the fields of IARP packet.

5) For updation of routing table in IARP:

(1) if packet arrived then_extract packet

Else

Send interrupt msg as \{ dest,mask,metric $\}$ and set next_hop_1 $1=$ destination

If type (intrpt) $=$ ("neighbour found")

Best_route=get_shortest_route $($ intrazone_routing table,d)

Set hop_count $=1$

Else

hop_count= infinity.

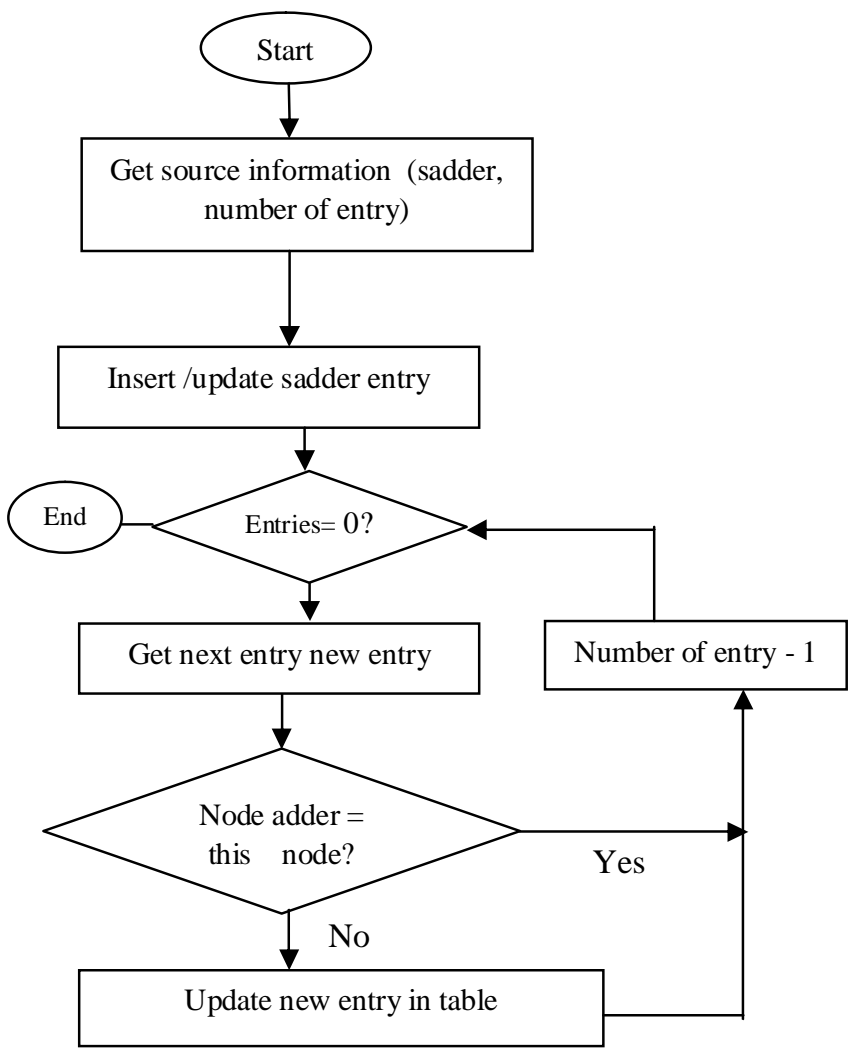

Figure9: IARP message sending part 2 


\subsection{Interzone Routing Protocol algorithm and steps:}

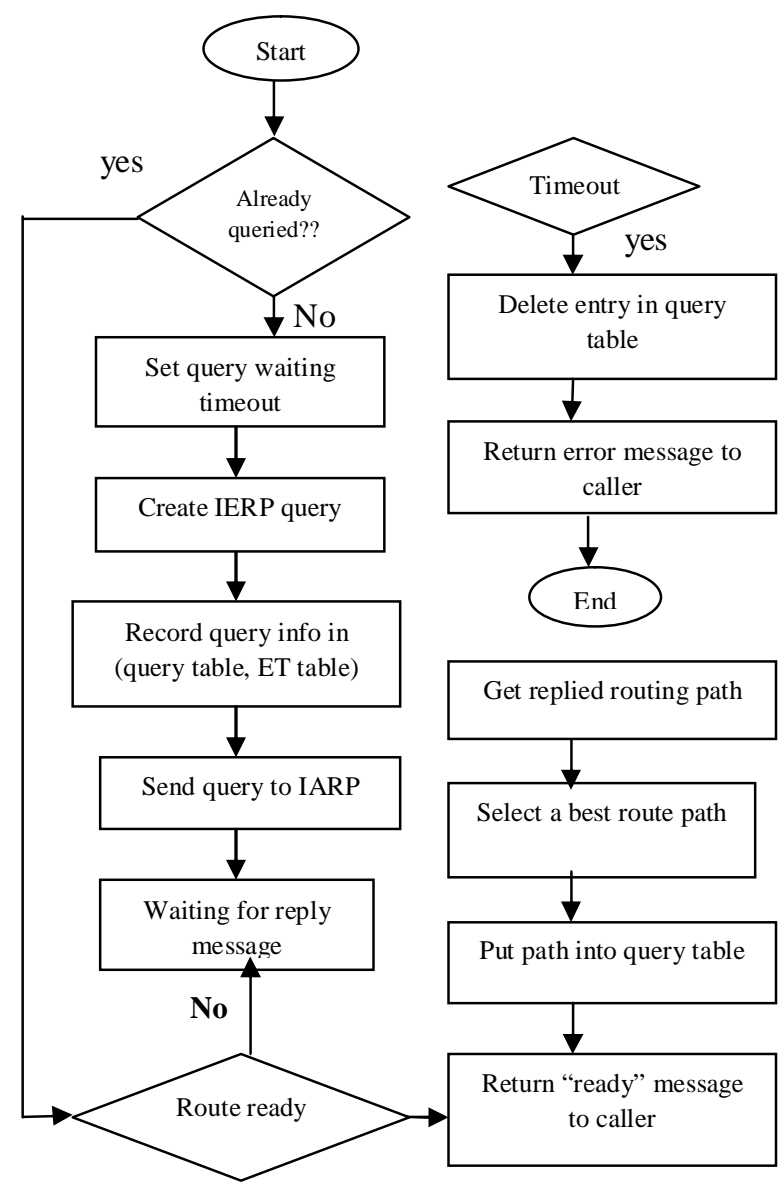

Figure10: IERP message sending

1) Create nodes and network between them.

2) Initialize them and start network.

3) Send packet from one node to another.

4) Receiving node extract packet and then load.

(a) Extract the fields of IERP packet to the following variables: \{link source, link dest, pkt_source, state_id, radius, flag, mask, link_metric

(b) load packet:Load the variables of aforementioned variables into the fields of IERP packet.

5) Update routing table

(a) if packet arrived then extract packet and set my_link_shange to false.

Else

(b) set $\{$ link_dest, mask, link_metric\} As interrupt Where

Link_source $=$ my_id

Pkt_source $=$ my_id

State_id $=$ my_link_state_id

Radius $=$ my_routing_zone_radius
If (type interrupt) == "neighbour found"

Link status $=$ up

Else

link_status $=$ DOWN

My_link_changed $=$ true

6) Route Zone node lost option

Interrupt $\rightarrow$ \{lost_host $\}$

Repair_link = false

For host and route

If lost host exist in route

Then set

Repair link $=$ true

Else

Remove(interzone routing table route $(\mathrm{m}))$

\subsection{Simulation Result}

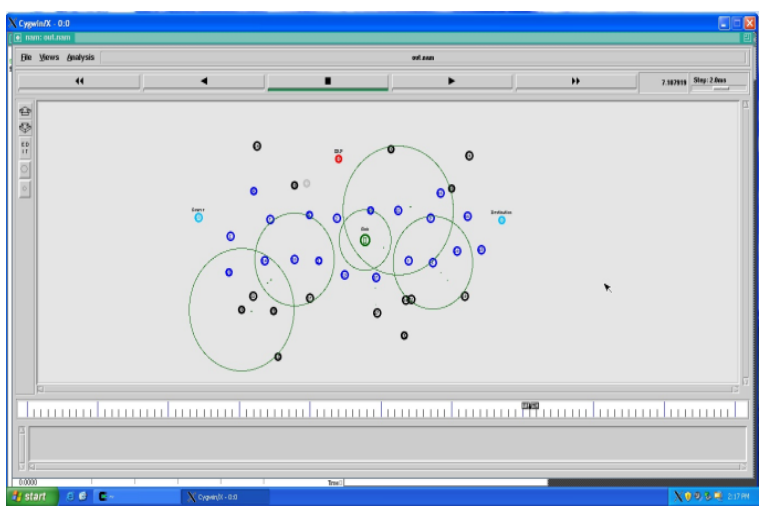

Figure11: Creation of Zone Radius \& transfer of data from $S$ to $D$

In the simulation we have taken approx 40 nodes used for communication between two nodes. In the Figure11 one can see the number of nodes and the link used between the nodes for communication is duplex-link and speed assigned between the nodes are 5 mbps. The time taken for sending and receiving is up to 10 milliseconds. We have taken Zone Radius $r=2$, but it varies in different zones.

The simulation is performed in NS-2 on windows platform through Cygwin, Nodes are deployed in the deployment area of $1485 * 700$. The complete deployment area is divided into zones. First of all, the Sender send the route request, it is to be determined with in the zone and if not get the appropriate response then it is to be forwarded to the peripheral nodes which then finds the destination in their zone and so on. When the appropriate node finds then route reply is to be send to the Sender. There is a Sink node which is dynamically assigned to 
each Zone. The Sink node collects the data and transfer it through the wireless nodes.(illustrated in Figure11 and Figure12)

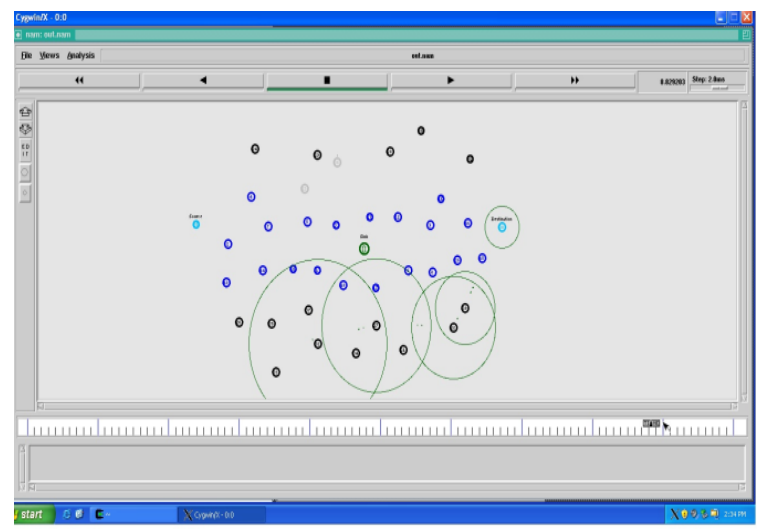

Figure 12: Transfer of data through different Zones

\section{Analysis of algorithm}

The throughput is shown in the red line, green line for the packet delivery ratio and blue line for packet delay. The graph shows (Figure13) that the packet delivery ratio and throughput increases continuously, with time as the number of packets increases on the network. When the traffic increases it declines but slowly. It is conspicuous that still packet loss ratio is very less. Delay becomes constant in the range of 5.0000 to 10.000 as the traffic increases on the network which $\mathrm{p}$ improves performance. So as compare to other dynamic protocols also like AODV, DSR $[11,12]$ etc its performance in terms of taken parameters such as throughput, packet delivery ratio and delay is much better.

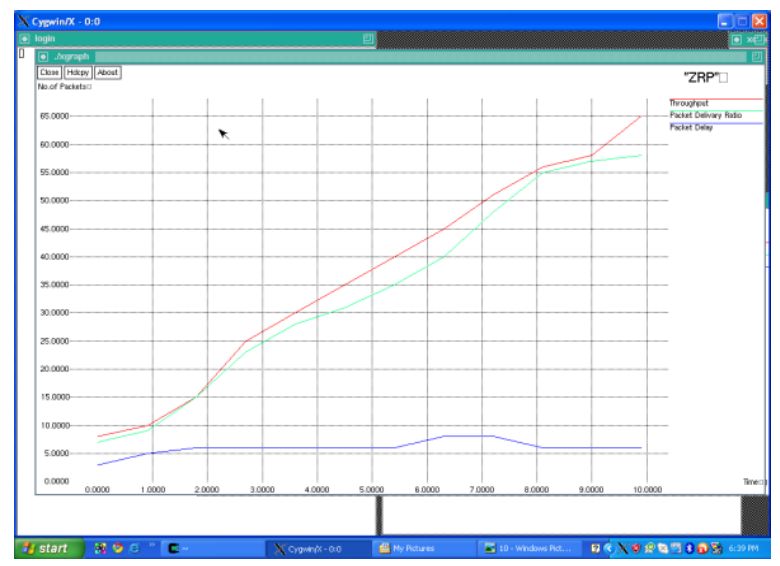

Figure13: Graph showing number of packets vs time

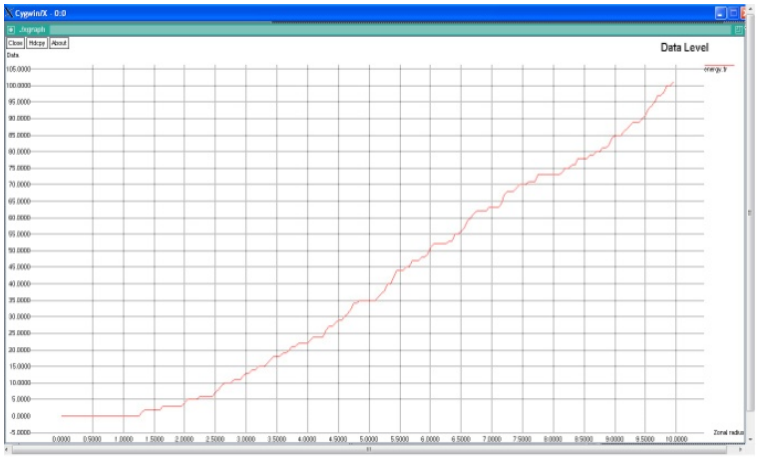

Fig14:Energy efficiency w.r.t zonal radius and traffic load

As we create different zones with the parameter zonal radius so energy is saved, and it is required w.r.t the zonal radius, lesser the number of zones cover by the packets lesser is the energy required. It is conspicuous from the graph that if the zonal radius is less and the destination resides there then lesser control information exchanges and lesser energy requires.

\section{Conclusion}

In this research work, I propose Zone Routing Protocol for the WSNs which over passes the features of its implementation in mobile ad-hoc networks. I recognized that ZRP gives better performance with WSNs rather than using mobile ad-hoc network. Specifically zones created in ZRP uses low energy and produces better throughput with good packet delivery ratio. I conclude from my implementation results that using ZRP with WSNs produce better results than theoretical analysis as well as the other proactive and reactive routing protocols. To summarize, I can say better throughput, packet delivery ratio, less overhead, delay is there. Also less control information is relayed to route discovery. As a result of which effective performance generated on the network.

\section{Future scope of Work}

It is interesting to see the performance of ZRP in large and realistic scenario. Also in future, we can work on required energy efficiency, security ,scalability, prolonged network life time and load balancing. I have provided the ZRP implementation used with WSNs which was earlier used with mobile ad-hoc network and the results achieved are much better than Ad hoc. So by applying hybrid routing in WSN, we can reduce the overhead, delay etc, further one can implement enhanced hybrid ZRP protocol to achieve better performance on the network. We can consider other parameters also, to improve the throughput. 


\section{References}

[1] Brijesh Patel and Sanjay Srivastava Dhirubhai Ambani Institute of Information and Communication Technology Gandhinagar 382 007, India "Performance Analysis of Zone Routing Protocols in Mobile Ad Hoc Networks" Communications (NCC), 2010 National Conference on Digital Object Identifier: 10.1109/NCC.2010.5430164 Publication Year: 2010 , Page(s): 1 - 5

[2] Yuki Sato*, Akio Koyama*, Leonard Barolli*** Department of Informatics, Graduate School of Science and Engineering,Yamagata University, Japan "A Zone Based Routing Protocol for Ad Hoc Networks and Its Performance Improvement by Reduction of Control Packets" Broadband, Wireless Computing, Communication and Applications (BWCCA), 2010 International Conference on Digital Object Identifier: 10.1109/BWCCA.2010.43 Publication Year: 2010 , Page(s): 17 - 24

[3] Nicklas Beijar Networking Laboratory, Helsinki University of Technology P.O. Box 3000, FIN-02015 HUT, Finland "Zone Routing Protocol (ZRP)"

[4] Prasun Sinha, Co-ordinated Sciences Laboratory, University of Illinois, Urbana Champaign "Scalable Unidirectional Routing with Zone Routing Protocol (ZRP) Extensions for Mobile Ad-Hoc Networks"

[5] Jan Schaumann "Analysis of the Zone Routing Protocol"

[6] Xin Liu, Quanyu Wang and Xuliang Jin Department of Computer Science and Technology Beijing Institute of Technology "An Energy-efficient Routing Protocol for Wireless Sensor Networks"

[7] Haruki Osanai Akio Koyama Department of Informatics, Graduate School of Science and Engineering, Yamagata University4-3-16 Jonan, Yonezawa, Japan "An Implementation and Evaluation of Zone-Based Routing Protocol for Mobile Ad-hoc Networks" Network-Based Information Systems (NBiS), 2011 14th International Conference on Digital Object Identifier: 10.1109/NBiS.2011.85 Publication Year: 2011 , Page(s): 517 $-522$

[8] Mohammed Ismail, @ Dr. M.Y. Sanavullah *Research Scholar, Department of Electronics, Vinayaka Mission University, Salem "Efficient On-Demand Routing Protocols to Optimize Network Coverage in Wireless Sensor Networks"

[9] Kamal BeydounLIFC, University of Franche-Comté "Energy-Efficient WSN Infrastructure"

[10] Fabian Nack Institute of Computer Science (ICS), Freie Universität Berlin "An Overview on Wireless Sensor Networks"

[11] P.Kuppusamy Dept. of Computer Science and Engineering Vivekananda College of Engineering for
Women Namakkal, India "A Study and Comparison of OLSR, AODV and TORA Routing Protocols in Ad Hoc Networks"

[12] SreeRangaRaju*, Jitendranath Mungara**,*Department of Telecommunication Engineering, Bangalore Institute of Technology Bangalore, India "ZRP Versus AODV and DSR: A Comprehensive Study on ZRP Performance Using Qualnet Simulator"

[13] K.P. Vijayakumar, P. Ganeshkumar and M. Anandaraj ,Department of IT, PSNA College of Engg. \& Tech., Dindigul, TamilNadu, India "Review on Routing Algorithms in Wireless Mesh Networks"

[14] Lotf, J.J.; Ghazani, S.H.H.N "Overview on routing protocols in wireless sensor networks" Computer Engineering and Technology (ICCET), 2010 2nd International Conference on Volume: 3 Digital Object Identifier: 10.1109/ICCET.2010.5485784 Publication Year: 2010 , Page(s): V3-610 - V3-614

[15] Changjiang Jiang; Min Xiang; Weiren Shi “ Overview of cluster-based routing protocols in wireless sensor networks " Electric Information and Control Engineering (ICEICE), 2011 International Conference on Digital Object Identifier: 10.1109/ICEICE.2011.5777573 Publication Year: 2011 , Page(s): 3414 - 3417 